describe the observed oxygen uptake curves under normal conditions, but it also predicts the hitherto inexplicable high value of the Hill constant (reflecting high haem-haem interaction) under conditions where the haemoglobin is dissociated. All the parameters in the equation can be evaluated by independent experiments, and the fit must therefore be seen as a strong argument in favour of the hypothesis.

The scheme demands differences in conformation of any chain when it combines with the ligand, and from dissociation behaviour and reactivity of sulphydryl groups Guidotti shows that there appear to be conformational differences between the deoxygenated dimer $a \beta$, the oxygenated dimer $\alpha^{*} \beta^{*}$, and the former in its combination with the latter, i.e., as $\alpha \beta \alpha^{*} \beta^{*}$. Guidotti expresses the hope that X-ray analysis of oxy- and deoxyhaemoglobins at high resolution will reveal conformational differences, no matter how small, within the chains.

So far this hope has not been too well borne out. The latest X-ray study from Perutz's laboratory (Muirhead et al., J. Mol. Biol., 28, 117; 1967) reports the structure of human deoxyhaemoglobin at $5.5 \AA$ resolution. Detailed comparison is available only with horse oxyhaemoglobin, but it is clear that in terms of tertiary structure the two proteins are closely similar in all respects, including the relationship between the haem group and the peptide chain. The change in the relative orientation of the sub-units, which amounts to a considerable rotation, has been precisely characterized, and changes in the contacts between sub-units are noted. At only two points, one in the $\alpha$ and the other in the $\beta$ chain, are slight conformational differences observed, though these are interpreted with caution. How such small differences of conformation might be capable of accounting for the very considerable chemical differences observed by Guidotti remains in doubt. The possibility that these reactions are governed by ionization differences which do not significantly affect the conformation must be considered. Guidotti's mechanism of ligand binding at this stage, however, still carries considerable conviction.

\section{Chloroplast DNA}

\section{from our Cell Biology Correspondent}

Ever since the discovery that mitochondria and chloroplasts contain DNA, the enzymes for DNA replication and transcription and distinct machinery for synthesizing protein, there has been speculation that these organelles evolved from symbiotic bacteria and that they have retained at least partial genetic autonomy. If this is so, it is obviously important to discover just how much genetic autonomy they have. Which proteins in the plastids are coded for by local DNA and which by nuclear DNA ? In two papers in Biochem. Biophys. Res. Comm. (28, 598 and 604; 1967), Smillie and his collaborators report on the way in which the DNA in the chloroplasts of Euglena gracilis serves as a source of information.

Not surprisingly, given the evidence that plastid ribosomal RNA is distinct from cytoplasmic ribosomal RNA, Steele-Scott and Smillie find that ${ }^{32} \mathrm{P}$-labelled ribosomal RNA from Euglena chloroplasts hybridizes with chloroplast DNA but that the total ribosomal RNA from cells grown in the dark, which lack well developed chloroplasts, fails to hybridize. They interpret this as showing that the chloroplast DNA rather than the nuclear DNA codes for chloroplast ribosomal RNA. A rough estimate indicates that each chloroplast has of the order of $20-45$ ribosomal RNA cistrons and presumably the chloroplast ribosomal proteins are also coded for by the DNA of the organelles.

To test whether photosynthetic enzymes either in the stroma or bound to lamellae are synthesized in the chloroplast and, therefore, by implication, whether they are coded for by the chloroplast DNA, Smillie et al. had to use a much more indirect approach. They reasoned that because protein synthesis in plastids and bacteria but not in plant cytoplasm is inhibited by chloramphenicol, whereas cycloheximide inhibits protein synthesis in plant cytoplasm but not in bacteria, enzymes made in chloroplasts should be inhibited by chloramphenicol but not by cycloheximide. The inhibitors were fed to Euglena differentiating chloroplast. and the cell extracts were assayed for three bound proteins of the electron transfer system (ferredoxin-NADP-reductase, cytochrome-552 and cytochrome-561) and for two stromal enzymes (ribulose-1. 5-bisphosphate carboxylase and NADP-glyceraldehyde3-phosphate dehydrogenase). Chloramphenicol, but not cycloheximide, inhibited the synthesis of the stromal enzymes, which must therefore be supposed to be synthesized in the chloroplasts. Synthesis of the electron transfer proteins was also inhibited by chloramphenicol, and this implicates the chloroplast ribosomes as the site of synthesis. In this case, however, the result is less clear cut because cycloheximide also had some inhibitory effect. Smillie et al. suggest that this inhibition by cycloheximide may be caused by some secondary indirect effect of the inhibitor on the synthesis of some chloroplast protein in the cytoplasm.

Thus it appears that enzymes involved in photosynthetic reduction of carbon dioxide and electron transport in chloroplasts are made within the organelle and probably coded for by the chloroplast DNA. By contrast, soluble mitochondrial enzymes involved in the oxidation of carbon compounds to carbon dioxide (Roodyn et al., 1962; Clark-Waller and Linnare, 1967) and the mitochondrial cytochrome $C$ (Beattie et al., 1966; Huang et al., 1966) are synthesized outside the mitochondria. These results suggest that a wider range of proteins is synthesized within chloroplasts than in mitochondria and therefore that chloroplasts have greater genetic autonomy than mitochondria.

\section{Chromatography and Spectroscopy \\ from G. R. Primavesi}

Almost from the beginning of gas chromatography. innumerable chromatographers and spectroscopists have collaborated in establishing the purity of standards and in identifying unknown components, but the first joint meeting of the Gas Chromatography and Infrared Discussion Groups of the Institute of Petroleum was held at Loughborough University of Technology on September 29.

A. R. Philpotts introduced the discussion with a quantitative estimate of the natural and technical limits of present techniques. Assuming that weak bands are just to be detected and that the whole of a sample can be put at the focus of an ordinary spectro- 